\title{
Quintessence and phantom cosmology with non-minimal derivative coupling
}

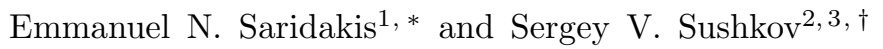 \\ ${ }^{1}$ Department of Physics, University of Athens, GR-15771 Athens, Greece \\ ${ }^{2}$ Department of General Relativity and Gravitation, \\ Kazan State University, Kremlevskaya str. 18, Kazan 420008, Russia \\ ${ }^{3}$ Department of Mathematics, Tatar State University of Humanities and Education, \\ Tatarstan str. 2, Kazan 420021, Russia
}

\begin{abstract}
We investigate cosmological scenarios with a non-minimal derivative coupling between the scalar field and the curvature, examining both the quintessence and the phantom cases in zero and constant potentials. In general, we find that the universe transits from one de Sitter solution to another, determined by the coupling parameter. Furthermore, according to the parameter choices and without the need for matter, we can obtain a Big Bang, an expanding universe with no beginning, a cosmological turnaround, an eternally contracting universe, a Big Crunch, a Big Rip avoidance and a cosmological bounce. This variety of behaviors reveals the capabilities of the present scenario.
\end{abstract}

\section{INTRODUCTION}

The cosmological research of the last three decades has elevated the role of scalar fields in the description of various sides of nature. Introduced as the driving mechanism for almost all inflation realizations [1], the dynamics of scalar fields gained new interest after observations provided indications for an accelerated universe expansion [2]. In particular, the new concept of "dark energy" was easier to be described by an extra scalar field dubbed quintessence [3], than with the traditional cosmological constant [4, 5], and the corresponding cosmological behavior proves to be much richer.

However, although the general belief is that the data are far from being conclusive, some data analyses suggested that the cosmological constant boundary, that is the phantom divide, has been crossed in the near cosmological past [6]. The simplest way to explain this unexpected behavior is the use of a phantom scalar field instead of a canonical one, that is a scalar with a negative sign of the kinetic term in the Lagrangian [7]. Although the discussion about the construction of quantum field theory of phantoms is still open in the literature (see for instance [8] for the causality and stability problems of phantom fields, but also [9] for attempts in constructing a phantom theory consistent with the basic requirements of quantum field theory, with the phantom fields arising as an effective description), the richness and capabilities of phantom cosmology has gained significant interest in the literature, extended apart from dark energy to inflation area too [10].

Apart from the aforementioned basic use of scalars (canonical or phantom ones), cosmological models where the fields are non-minimally coupled to gravity [11, 12] have been shown to present significant cosmological features, both for inflation and dark energy areas, and have been widely studied [13]. Additionally, one can further

*Electronic address: msaridak@phys.uoa.gr

${ }^{\dagger}$ Electronic address: sergey’sushkov@mail.ru extend these "scalar-tensor" theories, allowing for nonminimal couplings between the derivatives of the scalar fields and the curvature [14], and these scenarios reveal interesting cosmological behaviors [15]. ${ }^{1}$

In our recent work [18] we examined the cosmological scenario of a quintessence field with non-minimal derivative coupling, and we extracted exact solutions in the case of zero potential. In the present work we are interested in extending this analysis in the case of non-zero potentials, and furthermore, for completeness, to perform it for both a quintessence and a phantom field. The plan of the manuscript is as follows: In section $\amalg$ we construct the scenario and we extract the cosmological equations. In section [II we examine specific potential choices and we investigate the corresponding cosmological solutions for various parameter choices. Finally, in section IV we discuss the physical implications of the different universe evolutions, and we summarize the obtained results.

\section{COSMOLOGY WITH NON-MINIMAL DERIVATIVE COUPLING}

In this section we present the cosmological paradigm with non-minimal derivative coupling between a scalar field and the curvature. In order to describe the quintessence and the phantom field in a unified way we adopt the $\varepsilon$-notation, that is the parameter $\varepsilon$ takes the value +1 for the canonical field and -1 for the phantom one.

\section{A. Action and field equations}

Let us construct a gravitational theory of a scalar field $\phi$ with non-minimal derivative couplings to the curva-

\footnotetext{
${ }^{1}$ It is also worth mentioning a series of papers devoted to a nonminimal modification of the Einstein-Yang-Mills-Higgs theory [16] (see also the review [17] and references therein).
} 
ture. In general one could have various forms of such couplings. For instance in the case of four derivatives one could have the terms $\kappa_{1} R \phi_{, \mu} \phi^{, \mu}, \kappa_{2} R_{\mu \nu} \phi^{, \mu} \phi^{, \nu}, \kappa_{3} R \phi \square \phi$, $\kappa_{4} R_{\mu \nu} \phi \phi^{; \mu \nu}, \kappa_{5} R_{; \mu} \phi \phi^{, \mu}$ and $\kappa_{6} \square R \phi^{2}$, where the coefficients $\kappa_{1}, \ldots, \kappa_{6}$ are coupling parameters with dimensions of length-squared. However, as it was discussed in [14, 15, 18], using total divergencies and without loss of generality one can keep only the first two terms. Thus, the action for the cosmological scenarios at hand writes:

$S=\int d^{4} x \sqrt{-g}\left\{\frac{R}{8 \pi}-\left[\varepsilon g_{\mu \nu}+\kappa G_{\mu \nu}\right] \phi^{, \mu} \phi^{, \nu}-2 V(\phi)\right\}$,

where $V(\phi)$ is a scalar field potential, $g_{\mu \nu}$ is a metric, $g=$ $\operatorname{det}\left(g_{\mu \nu}\right), R$ is the scalar curvature, $G_{\mu \nu}$ is the Einstein tensor, and $\kappa$ is the single derivative coupling parameter with dimensions of length-squared.

Varying the action (11) with respect to the metric $g_{\mu \nu}$ leads to the gravitational field equations

$$
G_{\mu \nu}=8 \pi\left[\varepsilon T_{\mu \nu}+\kappa \Theta_{\mu \nu}\right]-8 \pi g_{\mu \nu} V(\phi),
$$

with

$$
\begin{aligned}
T_{\mu \nu}= & \nabla_{\mu} \phi \nabla_{\nu} \phi-\frac{1}{2} g_{\mu \nu}(\nabla \phi)^{2}, \\
\Theta_{\mu \nu}= & -\frac{1}{2} \nabla_{\mu} \phi \nabla_{\nu} \phi R+2 \nabla_{\alpha} \phi \nabla_{(\mu} \phi R_{\nu)}^{\alpha} \\
& +\nabla^{\alpha} \phi \nabla^{\beta} \phi R_{\mu \alpha \nu \beta}+\nabla_{\mu} \nabla^{\alpha} \phi \nabla_{\nu} \nabla_{\alpha} \phi \\
& -\nabla_{\mu} \nabla_{\nu} \phi \square \phi-\frac{1}{2}(\nabla \phi)^{2} G_{\mu \nu} \\
& +g_{\mu \nu}\left[-\frac{1}{2} \nabla^{\alpha} \nabla^{\beta} \phi \nabla_{\alpha} \nabla_{\beta} \phi+\frac{1}{2}(\square \phi)^{2}\right. \\
& \left.-\nabla_{\alpha} \phi \nabla_{\beta} \phi R^{\alpha \beta}\right] .
\end{aligned}
$$

Similarly, variation of the action (1) with respect to $\phi$ provides the scalar field equation of motion:

$$
\left[\varepsilon g^{\mu \nu}+\kappa G^{\mu \nu}\right] \nabla_{\mu} \nabla_{\nu} \phi=V_{\phi}
$$

where $V_{\phi} \equiv d V(\phi) / d \phi$.

\section{B. Cosmological equations}

Throughout this work we consider a spatially-flat background geometry with a metric

$$
d s^{2}=-d t^{2}+e^{2 \alpha(t)} d \mathbf{x}^{2}
$$

where $a(t) \equiv e^{\alpha(t)}$ is the scale factor, and $d \mathbf{x}^{2}$ is the Euclidian metric. Thus, the Hubble parameter is simply $H(t) \equiv \dot{a}(t) / a(t)=\dot{\alpha}(t)$.

As usual we assume a homogenous scalar field, namely $\phi=\phi(t)$. In this case the field equations (2) and (3) are reduced to the following system:

$$
\begin{aligned}
& 3 \dot{\alpha}^{2}=4 \pi \dot{\phi}^{2}\left(\varepsilon-9 \kappa \dot{\alpha}^{2}\right)+8 \pi V(\phi), \\
& -2 \ddot{\alpha}-3 \dot{\alpha}^{2}=4 \pi \dot{\phi}^{2}\left[\varepsilon+\kappa\left(2 \ddot{\alpha}+3 \dot{\alpha}^{2}+4 \dot{\alpha} \ddot{\phi} \dot{\phi}^{-1}\right)\right]- \\
& -8 \pi V(\phi), \\
& \varepsilon(\ddot{\phi}+3 \dot{\alpha} \dot{\phi})-3 \kappa\left(\dot{\alpha}^{2} \ddot{\phi}+2 \dot{\alpha} \ddot{\alpha} \dot{\phi}+3 \dot{\alpha}^{3} \dot{\phi}\right)=V_{\phi},
\end{aligned}
$$

where a dot denotes a derivative with respect to time. Note that equations (6) and (7) are of second order, while (5) is a first-order differential constraint for $\alpha(t)$ and $\phi(t)$.

The constraint (5) can be rewritten as:

$$
\dot{\phi}^{2}=\frac{3 \dot{\alpha}^{2}-8 \pi V(\phi)}{4 \pi\left(\varepsilon-9 \kappa \dot{\alpha}^{2}\right)}
$$

or equivalently as

$$
\dot{\alpha}^{2}=\frac{4 \pi \varepsilon \dot{\phi}^{2}+8 \pi V(\phi)}{3\left(1+12 \pi \kappa \dot{\phi}^{2}\right)}
$$

Therefore, as long as the parameters $\varepsilon$ and $\kappa$ and the potential $V(\phi)$ are given, the above relations provide restrictions for the possible values of $\dot{\alpha}$ and $\dot{\phi}$, since they have to give rise to non-negative $\dot{\phi}^{2}$ and $\dot{\alpha}^{2}$, respectively.

Let us now separate the equation for $\phi$ and $\alpha$. For this aim we resolve equations (6) and (7) with respect to $\ddot{\alpha}$ and $\ddot{\phi}$ and, using the relations (8) and (9), we eliminate $\dot{\phi}$ and $\dot{\alpha}$ from respective equations. We easily result to:

$$
\begin{gathered}
\ddot{\phi}=\frac{-2 \sqrt{3 \pi} \dot{\phi}\left[\varepsilon+\varepsilon 8 \pi \kappa \dot{\phi}^{2}-8 \pi \kappa V(\phi)\right] \sqrt{\left[\varepsilon \dot{\phi}^{2}+2 V(\phi)\right]\left(12 \pi \kappa \dot{\phi}^{2}+1\right)}+\left(12 \pi \kappa \dot{\phi}^{2}+1\right)\left(4 \pi \kappa \dot{\phi}^{2}+1\right) V_{\phi}}{\varepsilon\left(1+12 \pi \kappa \dot{\phi}^{2}+96 \pi^{2} \kappa^{2} \dot{\phi}^{4}\right)+8 \pi \kappa V(\phi)\left(12 \pi \kappa \dot{\phi}^{2}-1\right)} \\
\ddot{\alpha}=\frac{-\left(\varepsilon-3 \kappa \dot{\alpha}^{2}\right)\left(\varepsilon-9 \kappa \dot{\alpha}^{2}\right)\left[3 \dot{\alpha}^{2}-8 \pi V(\phi)\right]-4 \sqrt{\pi} \kappa \dot{\alpha} \sqrt{\left(\varepsilon-9 \kappa \dot{\alpha}^{2}\right)\left[3 \dot{\alpha}^{2}-8 \pi V(\phi)\right]} V_{\phi}}{1-9 \varepsilon \kappa \dot{\alpha}^{2}+54 \kappa^{2} \dot{\alpha}^{4}-8 \pi \kappa V(\phi)\left(\varepsilon+9 \kappa \dot{\alpha}^{2}\right)}
\end{gathered}
$$

We mention however that although the $\phi$-equation does

not contains $\alpha$-terms, the $\alpha$-equation in general contains 
$\phi$-terms arising from the potential $V(\phi)$.

\section{COSMOLOGICAL SCENARIOS AND SOLUTIONS}

In this section we examine specific cosmological scenarios, that is we consider specific potential choices.

\section{A. Zero potential: $V(\phi) \equiv 0$}

The case of a canonical field under zero potential has been investigated in [18]. Thus, in this subsection we restrict ourselves in the case of a phantom field, that is $\varepsilon=-1$. The field equations (5)-(7) now read

$$
\begin{aligned}
& 3 \dot{\alpha}^{2}=-4 \pi \dot{\phi}^{2}\left(1+9 \kappa \dot{\alpha}^{2}\right) \\
& -2 \ddot{\alpha}-3 \dot{\alpha}^{2}=-4 \pi \dot{\phi}^{2}\left[1-\kappa\left(2 \ddot{\alpha}+3 \dot{\alpha}^{2}+4 \dot{\alpha} \ddot{\phi} \dot{\phi}^{-1}\right)\right] \\
& \ddot{\phi}+3 \dot{\alpha} \dot{\phi}+3 \kappa\left[\dot{\alpha}^{2} \ddot{\phi}+2 \dot{\alpha} \ddot{\alpha} \dot{\phi}+3 \dot{\alpha}^{3} \dot{\phi}\right]=0
\end{aligned}
$$

The constraint (12) can be rewritten as:

$$
\dot{\phi}^{2}=-\frac{3 \dot{\alpha}^{2}}{4 \pi\left(1+9 \kappa \dot{\alpha}^{2}\right)},
$$

or equivalently as

$$
\dot{\alpha}^{2}=-\frac{4 \pi \dot{\phi}^{2}}{3\left(1+12 \pi \kappa \dot{\phi}^{2}\right)} .
$$

From these relations we deduce that $\dot{\alpha}$ and $\dot{\phi}$ should obey the following conditions:

$$
\begin{gathered}
1+9 \kappa \dot{\alpha}^{2} \leq 0 \\
1+12 \pi \kappa \dot{\phi}^{2} \leq 0
\end{gathered}
$$

Note that these conditions are only fulfilled for $\kappa<0$. Assuming $\kappa=-k^{2}$, we find

$$
\begin{gathered}
\dot{\alpha}^{2} \geq \frac{1}{9 k^{2}} \\
\dot{\phi}^{2} \geq \frac{1}{12 \pi k^{2}} .
\end{gathered}
$$

The separate $\phi$ and $\alpha$-equations (10) and (11) respectively) read:

$$
\begin{gathered}
\ddot{\phi}=-\frac{2 \sqrt{3 \pi} \dot{\phi}^{2}\left(8 \pi \kappa \dot{\phi}^{2}-1\right) \sqrt{12 \pi \kappa \dot{\phi}^{2}-1}}{1-12 \pi \kappa \dot{\phi}^{2}+96 \pi^{2} \kappa^{2} \dot{\phi}^{4}} \\
\ddot{\alpha}=-\frac{3 \dot{\alpha}^{2}\left(3 k^{2} \dot{\alpha}^{2}-1\right)\left(9 k^{2} \dot{\alpha}^{2}-1\right)}{1-9 k^{2} \dot{\alpha}^{2}+54 k^{4} \dot{\alpha}^{4}} .
\end{gathered}
$$

From these equations we deduce that the case at hand exhibits three qualitatively different cases:

A1. $\left(9 k^{2}\right)^{-1}<\dot{\alpha}^{2}<\left(3 k^{2}\right)^{-1}$. In this case $\ddot{\alpha}$ is positive, and $\dot{\alpha}$ increases with time. The solution $\alpha$ is varying between two de Sitter asymptotics: $\alpha_{t \rightarrow-\infty}=t / 3 k$ and $\alpha_{t \rightarrow \infty}=t / \sqrt{3} k$.

A2. $\dot{\alpha}^{2}=\left(3 k^{2}\right)^{-1}, \ddot{\alpha}=0$. One has exactly the de Sitter solution

$$
\alpha(t)=t / \sqrt{3} k, \quad \phi(t)=t / \sqrt{8 \pi} k .
$$

A3. $\dot{\alpha}^{2}>\left(3 k^{2}\right)^{-1}$. In this case $\ddot{\alpha}$ is negative, and $\dot{\alpha}$ decreases with time. Nevertheless, we remind that $\dot{\alpha}$ remains larger than $1 / \sqrt{3} k$ always. The solution $\alpha(t)$ is varying between two asymptotics. The $t \rightarrow \infty$ asymptotic is the de Sitter one: $\alpha_{t \rightarrow \infty}=t / \sqrt{3} k$. The second asymptotic can be obtained as follows: Assuming $\dot{\alpha} \rightarrow \infty$ at $t \rightarrow t_{i}$ gives the following asymptotical form of Eq. (22):

$$
\begin{aligned}
& \ddot{\alpha} \approx-\frac{3}{2} \dot{\alpha}^{2} \\
& \dot{\phi}^{2}=\frac{1}{12 \pi k^{2}},
\end{aligned}
$$

with the asymptotic solution

$$
\begin{aligned}
& \alpha_{t \rightarrow t_{i}}=\alpha_{i}+\frac{2}{3} \ln \left(t-t_{i}\right) \\
& \phi_{t \rightarrow t_{i}}=\phi_{i}+\frac{1}{2 \sqrt{3} \pi k}\left(t-t_{i}\right) .
\end{aligned}
$$

Thus, by construction, the moment $t=t_{i}$ corresponds to a cosmological singularity.

In order to present this cosmological behavior more transparently, we perform a numerical elaboration of the model at hand, namely of equation (22), and the results are presented in Fig. 1. We mention that the possible

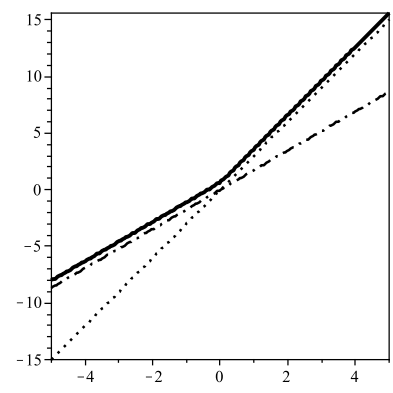

(a)

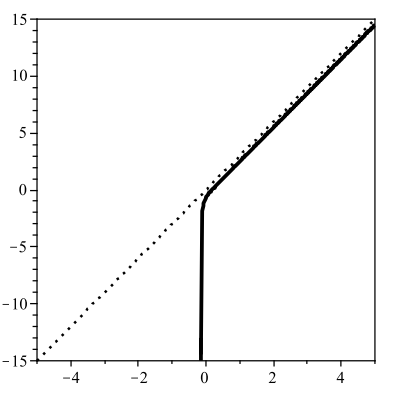

(b)
FIG. 1: The evolution of $\alpha(t)$ in the phantom case with zero potential. The dash-dotted and dotted lines denote the de Sitter asymptotics $t / 3 k$ and $t / \sqrt{3} k$, respectively. The solid lines corresponds to $\alpha(t)$ with (a) $\left(9 k^{2}\right)^{-1}<\dot{\alpha}^{2}<\left(3 k^{2}\right)^{-1}$ (case A1); (b) $\dot{\alpha}^{2}>\left(3 k^{2}\right)^{-1}$ (case A3). We have considered $k^{2}=\frac{1}{27}$.

singular behavior of $\alpha(t)$ means that the scale factor itself $\left(a(t)=e^{\alpha(t)}\right)$ starts from zero at some initial time. 


\section{B. Cosmological constant: $V(\phi) \equiv \Lambda=$ const}

In this particular scenario the separate $\phi$ and $\alpha$ equations (10) and (11) read:

$$
\begin{gathered}
\ddot{\phi}=\frac{-\sqrt{12 \pi} \dot{\phi} \sqrt{\varepsilon \dot{\phi}^{2}+2 \Lambda} \sqrt{12 \pi \kappa \dot{\phi}^{2}+1}\left[\varepsilon+8 \pi \varepsilon \kappa \dot{\phi}^{2}-8 \pi \kappa \Lambda\right]}{\varepsilon\left(1+12 \pi \kappa \dot{\phi}^{2}+96 \pi^{2} \kappa^{2} \dot{\phi}^{4}\right)+8 \pi \kappa \Lambda\left(12 \pi \kappa \dot{\phi}^{2}-1\right)} \\
\ddot{\alpha}=\frac{3\left(\varepsilon_{\Lambda} H_{\Lambda}^{2}-\dot{\alpha}^{2}\right)\left(\varepsilon-3 \kappa \dot{\alpha}^{2}\right)\left(\varepsilon-9 \kappa \dot{\alpha}^{2}\right)}{1-9 \varepsilon \kappa \dot{\alpha}^{2}+54 \kappa^{2} \dot{\alpha}^{4}-3 \kappa \varepsilon_{\Lambda} H_{\Lambda}^{2}\left(\varepsilon+9 \kappa \dot{\alpha}^{2}\right)},
\end{gathered}
$$

where for simplicity we have defined $H_{\Lambda}=\sqrt{8 \pi|\Lambda| / 3}$ and $\varepsilon_{\Lambda}=\operatorname{sign} \Lambda$. It proves convenient to consider separately the various cases arising from specific choices of the parameters $\varepsilon, \Lambda$, and $\kappa$, and in the following we present the eight qualitatively different cases of the scenario at hand.

B1. $\varepsilon=1, \Lambda>0, \kappa>0$.

It is easy to see that the $\alpha$-equation (28) has three trivial particular solutions: (i) $\alpha(t)=H_{\Lambda} t$, (ii) $\alpha(t)=$ $t / \sqrt{3 \kappa}$, and (iii) $\alpha(t)=t / \sqrt{9 \kappa}$. Sequentially, substituting them into the whole system of field equations (5)-(7), one may straightforwardly find the following exact solutions:

$$
\begin{aligned}
& \alpha(t)=H_{\Lambda} t, \quad \phi(t) \equiv \phi_{0}=\text { const }, \\
& \alpha(t)=\frac{t}{\sqrt{3 \kappa}}, \quad \phi(t)=\sqrt{\frac{3 \kappa H_{\Lambda}^{2}-1}{8 \pi \kappa}} t, \quad H_{\Lambda} \geq \frac{1}{\sqrt{3 \kappa}},
\end{aligned}
$$

which describe de Sitter universes. However, the third solution $\alpha(t)=t / \sqrt{9 \kappa}$ cannot in general satisfy equations (5)-(7) (apart from the fine-tuned case $1 / \sqrt{9 \kappa}=H_{\Lambda}$ ).

More generally, the constraint (8) gives the following restrictions for $\dot{\alpha}^{2}$ :

$$
x_{1}<\dot{\alpha}^{2}<x_{2},
$$

where $x_{1}=\min \left(1 / 9 \kappa, H_{\Lambda}^{2}\right)$ and $x_{2}=\max \left(1 / 9 \kappa, H_{\Lambda}^{2}\right)$. The second derivative $\ddot{\alpha}$, given by relation (28), is negative if $H_{\Lambda}^{2}<1 / 9 \kappa$, and positive if $H_{\Lambda}^{2}>1 / 9 \kappa$, and thus $\dot{\alpha}$ is decreasing or increasing with time. As it is deduced, the corresponding solutions for $\alpha(t)$ are varying between the two de Sitter asymptotics depending on values of parameters $H_{\Lambda}$ and $\kappa$. This behavior can be observed in Fig. 2, arisen from numerical elaboration.

B2. $\varepsilon=1, \Lambda>0, \kappa<0$.

In this case equations (5)-(7) have the de Sitter solution $\alpha(t)=H_{\Lambda} t, \phi(t) \equiv \phi_{0}=$ const. Additionally, the constraint (8) yields

$$
\dot{\alpha}^{2}>H_{\Lambda}^{2}
$$

Under this condition $\ddot{\alpha}$, given by relation (28), is negative and $\dot{\alpha}$ is decreasing with time. The corresponding solution $\alpha(t)$ is singular at some initial moment of time, i.e., $\lim _{t \rightarrow t_{i}} \alpha(t)=-\infty$, while for large times $\alpha(t)$ tends to de Sitter asymptotic $H_{\Lambda} t$. This behavior can be seen in Fig. 3.

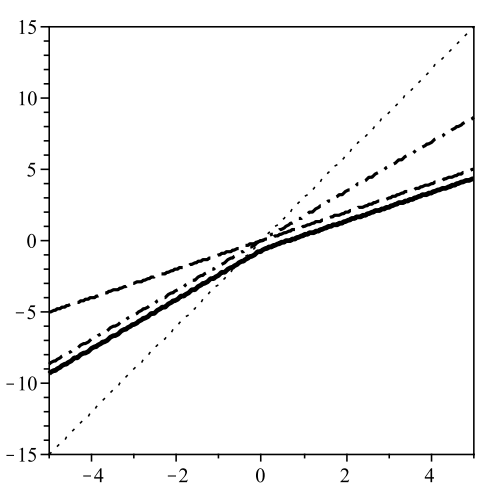

(a)

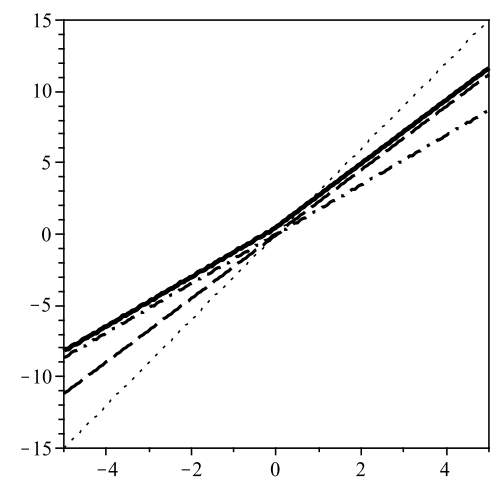

(b)

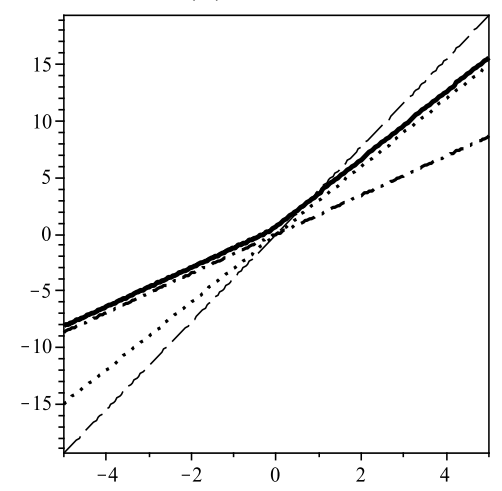

(c)

FIG. 2: The evolution for $\alpha(t)$ for $\varepsilon=1$ (quintessence), $\Lambda>0, \kappa>0$ (case B1). The dashed, dash-dotted, and dotted lines denote de Sitter asymptotics $\alpha(t)=H_{\Lambda} t, t / \sqrt{9 \kappa}$, and $t / \sqrt{3 \kappa}$, respectively. A solid line corresponds to $\alpha(t)$ with (a) $H_{\Lambda}^{2}<\dot{\alpha}^{2}<1 / 9 \kappa$; (b) $1 / 9 \kappa<\dot{\alpha}^{2}<H_{\Lambda}^{2}<1 / 3 \kappa$; (c) $1 / 9 \kappa<$ $\dot{\alpha}^{2}<1 / 3 \kappa<H_{\Lambda}^{2}$. In graph (a) we have considered $H_{\Lambda}^{2}=1$, in graph (b) $H_{\Lambda}^{2}=5$, and in graph (c) $H_{\Lambda}^{2}=10$; everywhere $\kappa=\frac{1}{27}$.

B3. $\varepsilon=-1, \Lambda>0, \kappa>0$.

For these parameter choices, equations (5)-(7) have the de Sitter solution $\alpha(t)=H_{\Lambda} t, \phi(t) \equiv \phi_{0}=$ const. Generally, the constraint (8) gives

$$
\dot{\alpha}^{2}<H_{\Lambda}^{2} \text {. }
$$

Under this restriction $\ddot{\alpha}$, from (28), is positive and $\dot{\alpha}$ is 


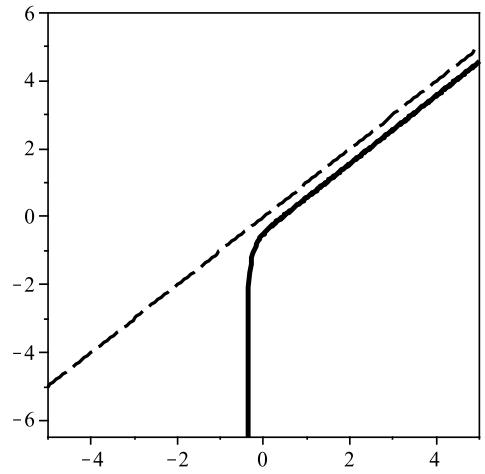

FIG. 3: The evolution for $\alpha(t)$ for $\varepsilon=1$ (quintessence), $\Lambda>0, \kappa<0$ (case B2). The dashed line corresponds to the de Sitter solution $\alpha(t)=H_{\Lambda} t$ with $H_{\Lambda}<1 / \sqrt{3|\kappa|}$. The solid curve corresponds to a solution with $\dot{\alpha}^{2}>H_{\Lambda}^{2}$. We have considered $H_{\Lambda}^{2}=1$ and $\kappa=-\frac{1}{27}$.

increasing with time. The corresponding solution for $\alpha(t)$ varies between two de Sitter asymptotics: $\alpha(t \rightarrow-\infty) \approx$ $-H_{\Lambda} t$ and $\alpha(t \rightarrow \infty) \approx H_{\Lambda} t$. This behavior is more transparently shown in Fig. 4 .

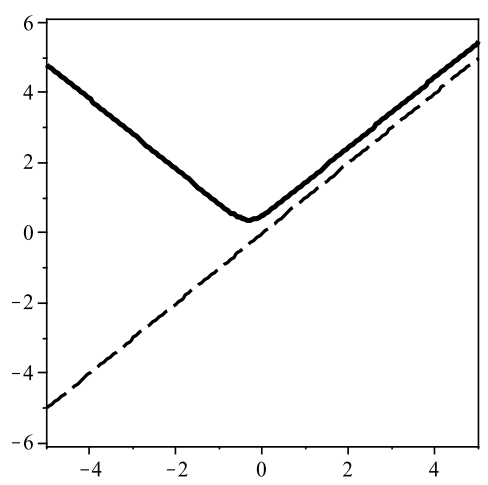

FIG. 4: The evolution for $\alpha(t)$ for $\varepsilon=-1$ (phantom), $\Lambda>$ $0, \kappa>0$ (case B3). The dashed line corresponds to the de Sitter solution $\alpha(t)=H_{\Lambda} t$ with $H_{\Lambda}<1 / \sqrt{3 \kappa}$. The solid line corresponds to a solution with $\dot{\alpha}^{2}<H_{\Lambda}^{2}$. We have considered $H_{\Lambda}^{2}=1$ and $\kappa=\frac{1}{27}$.

B4. $\varepsilon=-1, \Lambda>0, \kappa<0$.

In this case equations (5)-(7) possess two different de Sitter solutions:

$$
\begin{aligned}
& \alpha(t)=H_{\Lambda} t, \phi(t) \equiv \phi_{0}=\text { const } \\
& \alpha(t)=\frac{t}{\sqrt{3|\kappa|}}, \phi(t)=\sqrt{\frac{1-3|\kappa| H_{\Lambda}^{2}}{8 \pi|\kappa|}} t, H_{\Lambda} \leq \frac{1}{\sqrt{3|\kappa|}} .
\end{aligned}
$$

The constraint (8) now yields

$$
\dot{\alpha}^{2}<x_{1} \quad \text { or } \quad \dot{\alpha}^{2}>x_{2},
$$

where $x_{1}=\min \left(1 / 9 \kappa, H_{\Lambda}^{2}\right)$ and $x_{2}=\max \left(1 / 9 \kappa, H_{\Lambda}^{2}\right)$. Thus, the behavior of $\alpha(t)$ satisfying the above conditions depends on the specific values of the parameters
$H_{\Lambda}$ and $\kappa$. The corresponding possible types of solutions are demonstrated in Fig. 5 .

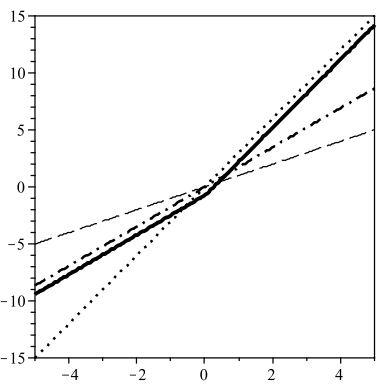

(a)

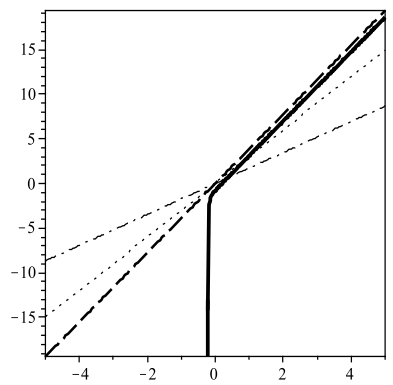

(c)

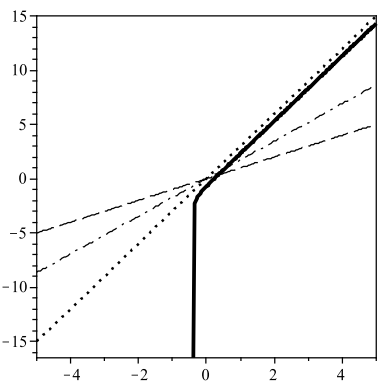

(b)

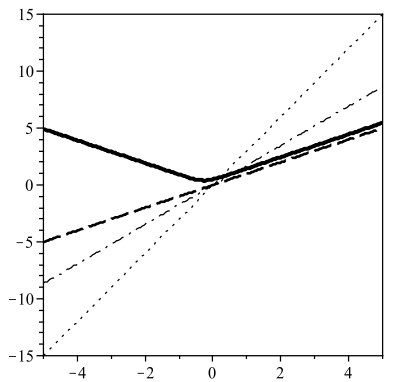

(d)

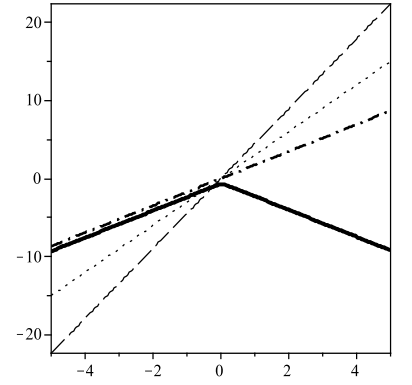

(e)

FIG. 5: The evolution of $\alpha(t)$ for $\varepsilon=-1$ (phantom), $\Lambda>0$, $\kappa<0$ (case B4). The dashed, dash-dotted, and dotted lines denote the de Sitter asymptotics $\alpha(t)=H_{\Lambda} t, t / \sqrt{9|\kappa|}$, and $t / \sqrt{3|\kappa|}$, respectively. The solid lines corresponds to $\alpha(t)$ with (a) $H_{\Lambda}^{2}<1 / 9|\kappa|<\dot{\alpha}^{2}<1 / 3|\kappa|$; (b) $H_{\Lambda}^{2}<1 / 9|\kappa|<1 / 3|\kappa|<$ $\dot{\alpha}^{2}$; (c) $1 / 3|\kappa|<H_{\Lambda}^{2}<\dot{\alpha}^{2}$; (d) $\dot{\alpha}^{2}<H_{\Lambda}^{2}<1 / 9|\kappa|$; (e) $\dot{\alpha}^{2}<$ $1 / 9|\kappa|<H_{\Lambda}^{2}$. In graphs (a), (b) and (d) we have considered $H_{\Lambda}^{2}=1$, in graphs (c) and (e) $H_{\Lambda}^{2}=15$; everywhere $\kappa=-\frac{1}{27}$.

B5. $\varepsilon=1, \Lambda<0, \kappa>0$.

In this case the constraint (8) leads to

$$
\dot{\alpha}^{2}<\frac{1}{9 \kappa} \text {. }
$$

Under this condition $\ddot{\alpha}$, from relation (28), is negative and $\dot{\alpha}$ is decreasing with time. The corresponding solution for $\alpha(t)$ varies between the two de Sitter asymptotics: $\alpha_{t \rightarrow-\infty} \approx t / \sqrt{9 \kappa}$ and $\alpha_{t \rightarrow \infty} \approx-t / \sqrt{9 \kappa}$. These features are presented in Fig. 6, arisen from numerical elaboration. 


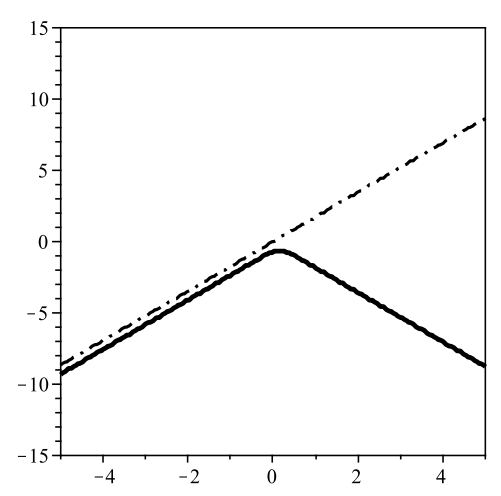

FIG. 6: The evolution of $\alpha(t)$ for $\varepsilon=1$ (quintessence), $\Lambda<0$, $\kappa>0$ (case B5). The dash-dotted line denotes the de Sitter asymptotic $\alpha(t)=t / \sqrt{9 \kappa}$. The solid curve corresponds to $\alpha(t)$ with $\dot{\alpha}^{2}<1 / 9 \kappa$. We have considered $H_{\Lambda}^{2}=1$ and $\kappa=\frac{1}{27}$.

B6. $\varepsilon=1, \Lambda<0, \kappa<0$.

For these parameter sub-class, the constraint (8) does not lead to any restriction on the values of $\dot{\alpha}^{2}$. Additionally, the second derivative $\ddot{\alpha}$, from (28), is negative and $\dot{\alpha}$ is decreasing with time. The corresponding solution for $\alpha(t)$ varies between the two singular solutions: $\alpha_{t \rightarrow \pm t^{*}}=-\infty$. This behavior can be seen more transparently in Fig. 7.

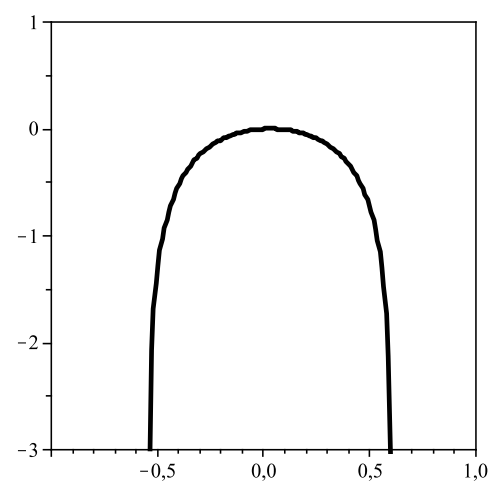

FIG. 7: The evolution of $\alpha(t)$ for $\varepsilon=1, \Lambda<0, \kappa<0$ (case B6). We have considered $H_{\Lambda}^{2}=1$ and $\kappa=-\frac{1}{27}$.

B7. $\varepsilon=-1, \Lambda<0, \kappa>0$.

In this particular case, the constraint (8) cannot be fulfilled. Therefore, there are no solutions corresponding to this scenario sub-class.

B8. $\varepsilon=-1, \Lambda<0, \kappa<0$.

In this case equations (5)-(7) posses the following de Sitter solution:

$$
\alpha(t)=\frac{t}{\sqrt{3|\kappa|}}, \quad \phi(t)=\sqrt{\frac{1+3|\kappa| H_{\Lambda}^{2}}{8 \pi|\kappa|}} t .
$$

The constraint (8) now gives

$$
\dot{\alpha}^{2}>\frac{1}{9|\kappa|} .
$$

Under this condition $\ddot{\alpha}$ from (28) is positive if $1 / 9|\kappa|<$ $\dot{\alpha}^{2}<1 / 3|\kappa|$, and negative if $\dot{\alpha}^{2}>1 / 3|\kappa|$; respectively, $\dot{\alpha}$ is increasing or decreasing with time. The two possible types of solutions for $\alpha(t)$ are presented in Fig. 8, arisen from numerical elaboration.

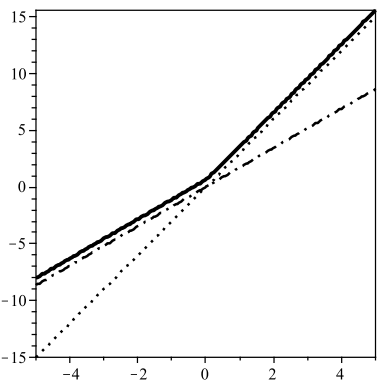

(a)

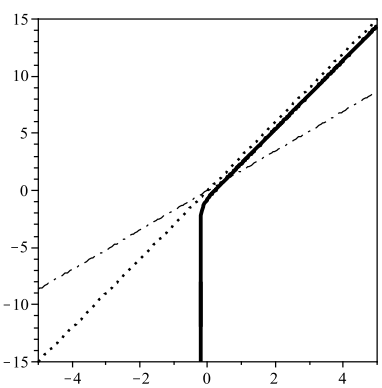

(b)
FIG. 8: The evolution of $\alpha(t)$ for $\varepsilon=-1$ (phantom), $\Lambda<0$, $\kappa<0$ (case B8). The dash-dotted, and dotted lines denote de Sitter asymptotics $\alpha(t)=t / \sqrt{9|\kappa|}$, and $t / \sqrt{3|\kappa|}$, respectively. The solid curve corresponds to $\alpha(t)$ with (a) $1 / 9|\kappa|<\dot{\alpha}^{2}<$ $1 / 3|\kappa| ;$ (b) $\dot{\alpha}^{2}>1 / 3|\kappa|$. We have considered $H_{\Lambda}^{2}=1$ and $\kappa=-\frac{1}{27}$.

We close this subsection by mentioning that for $\Lambda \rightarrow 0$, the scenario at hand coincides with the previously studied quintessence case with $V=0$ [18], or with the phantom with $V=0$ examined in the previous subsection.

Finally, note that in principle one could extend the aforementioned analysis to more complicated potentials, such as the exponential and the power-law one. Unfortunately, the complexity of the non-minimal derivative coupling does not allow for the extraction of any analytic solutions in these cases. The examination of the cosmological behavior of such scenarios must be based on numerical investigation, and this is left for a future investigation [19].

\section{DISCUSSION AND CONCLUSIONS}

In this work we investigated cosmological scenarios where there is a non-minimal derivative coupling between the scalar field and the curvature. In order to be complete, we considered both quintessence and phantom fields, although the later case could be ambiguous at the quantum level. Finally, in order to examine the pure effects of these scenarios, we have not included the matter content of the universe, although this can be straightforwardly taken into account.

A first observation is that the non-minimal derivative coupling leads to qualitatively different behavior, comparing to the uncoupled case, even for the simple cases 
of zero or constant potentials. In particular, as we observe the universe evolves between two asymptotic de Sitter solutions, characterized by the strength of the coupling. In the limit where the coupling tends to zero and in the cases where the solutions exist, these two asympotics coincide and the system acquires only one de Sitter solution, namely the one that is exhibited from the corresponding conventional (that is uncoupled) model of a universe without matter. We mention that the transition between the two de Sitter solutions is a pure effect of the non-minimal derivative coupling, and it does not require the presence and the role of matter. Finally, note that as time roll backwards, the scale factor of the universe $\left(a(t)=e^{\alpha(t)}\right)$ can be either eternally decreasing, or become zero at some initial time. Thus, our scenario exhibits either the Big Bang, or it corresponds to an eternally expanding universe with no beginning, with the later case arising easily, without the need of a specially designed potential as in conventional cosmology [20].

An additional feature of the scenario at hand is the radically different evolution of a quintessence universe in some solution sub-classes. In particular, for negative cosmological constant and positive coupling (case B5) the scale factor of the universe is growing, it reaches a maximum, and then it decreases. This is the realization of the cosmological turnaround, in which the universe transits from expansion to contraction [5, 21]. The fact that this is obtained solely from the dynamics of the non-minimal derivative coupling, without the need for matter or for exotic gravitational terms, makes the scenario at hand very interesting. Lastly, note that the contracting phase is eternal, that is the universe does not result to a Big Crunch [5].

In similar lines, in the quintessence case with negative cosmological constant and negative coupling (case B6), the scale factor starts from zero at some initial time and returns to zero at some final time. This is the realization of a universe starting from a Big Bang and ending with a Big Crunch [5], and the fact that this is obtained without the need of matter is a novel effect of the non-minimal derivative coupling.

The phantom evolution exhibits also the aforementioned behavior. Apart from an eternally expanding universe, including the transition between two de Sitter solutions, as we observe in Fig. 5(e) (case B4), that is for positive cosmological constant and negative coupling, the universe can experience the cosmological turnaround. This is radically different comparing to the uncoupled phantom scenarios, which not only cannot experience the turnaround, but on the contrary, in the absence of matter they result to a Big Rip 22]. It seems that the nonminimal derivative coupling smoothers or (for large coupling) completely alters the evolution, leaving phantom cosmology free of a Big Rip. This new and significant behavior reveals the richness of the scenario at hand.

However, the phantom case exhibits an additional surprising feature, that is not present in the quintessence scenario. As we observe in Fig. 4 (case B3, that is positive cosmological constant with positive coupling), as well as in Fig. 5(d) (case B4, that is positive cosmological constant with negative coupling), the universe can transit from the contracting to the expanding phase, without meeting any singularity. This is just the cosmological bounce [23], and its realization from a sole phantom field make the scenario at hand very interesting.

In summary, the paradigm of non-minimal derivative coupling either in the quintessence or in the phantom case, may have important cosmological implications, even in its simplified realization where matter is absent. Apart from the transition between different de Sitter solutions, according to the parameter choices we can obtain a Big Bang, an expanding universe with no beginning, a cosmological turnaround, an eternally contracting universe, a Big Crunch, a Big Rip avoidance and a cosmological bounce, and this variety of behaviors reveals the capabilities of the scenario. Furthermore, one could generalize this paradigm in the case where both the quintessence and the phantom fields are present, that is to generalize the so called "quintom" paradigm [24] in the case of nonminimal derivative coupling. In these scenarios one could obtain the combination of the above behaviors, such as to obtain a cyclic cosmology [25]. Definitely, this subject deserves further investigation.

\section{Acknowledgments}

This work was supported in part by the Russian Foundation for Basic Research grants No. 08-02-91307 and 08-02-00325.
[1] A. Linde, Particle Physics and Inflationary Cosmology (1990), Harwood Publ. London.

[2] A. G. Riess et al. [Supernova Search Team Collaboration], Astron. J. 116, 1009 (1998); S. Perlmutter et al. [Supernova Cosmology Project Collaboration], Astrophys. J. 517, 565 (1999); C. L. Bennett et al., Astrophys. J. Suppl. 148, 1 (2003); M. Tegmark et al. [SDSS Collaboration], Phys. Rev. D 69, 103501 (2004); S. W. Allen, et al., Mon. Not. Roy. Astron. Soc. 353, 457 (2004).
[3] B. Ratra and P. J. E. Peebles, Phys. Rev. D 37, 3406 (1988); C. Wetterich, Nucl. Phys. B 302, 668 (1988); A. R. Liddle and R. J. Scherrer, Phys. Rev. D 59, 023509 (1999); I. Zlatev, L. M. Wang and P. J. Steinhardt, Phys. Rev. Lett. 82, 896 (1999); Z. K. Guo, N. Ohta and Y. Z. Zhang, Mod. Phys. Lett. A 22, 883 (2007); S. Dutta, E. N. Saridakis and R. J. Scherrer, Phys. Rev. D 79, 103005 (2009).

[4] V. Sahni and A. Starobinsky, Int. J. Mod. Phy. D 9, 373 
(2000).

[5] P. J. E. Peebles and B. Ratra, Rev. Mod. Phys. 75, 559 (2003).

[6] D. Huterer and A. Cooray, Phys. Rev. D 71, 023506 (2005); B. Feng, X. L. Wang and X. M. Zhang, Phys. Lett. B 607, 35 (2005).

[7] R. R. Caldwell, Phys. Lett. B 545, 23 (2002); R. R. Caldwell, M. Kamionkowski and N. N. Weinberg, Phys. Rev. Lett. 91, 071301 (2003); S. Nojiri and S. D. Odintsov, Phys. Lett. B 562, 147 (2003); V. K. Onemli and R. P. Woodard, Phys. Rev. D 70, 107301 (2004); E. N. Saridakis, Nucl. Phys. B 819, 116 (2009).

[8] J. M. Cline, S. Jeon and G. D. Moore, Phys. Rev. D 70, 043543 (2004).

[9] S. Nojiri and S. D. Odintsov, Phys. Lett. B 562, 147 (2003); S. Nojiri and S. D. Odintsov, Phys. Lett. B 571, 1 (2003).

[10] P. F. Gonzalez-Diaz and J. A. Jimenez-Madrid, Phys. Lett. B 596, 16 (2004); S. Capozziello, S. Nojiri and S. D. Odintsov, Phys. Lett. B 632, 597 (2006).

[11] V. Sahni and S. Habib, Phys. Rev. Lett. 81, 1766 (1998); S. Sen and A. A. Sen, Phys. Rev. D 63, 124006 (2001); T. Chiba, Phys. Rev. D 60, 083508 (1999); F. Perrotta, C. Baccigalupi and S. Matarrese, Phys. Rev. D 61, 023507 (2000); E. Elizalde, S. Nojiri and S. Odintsov, Phys. Rev. D 70, 043539 (2004); V. K. Onemli and R. P. Woodard, Phys. Rev. D 70, 107301 (2004); E. Elizalde et al, Phys. Rev. D 77, 106005 (2008).

[12] V. Faraoni, Phys. Rev. D 68, 063508 (2003); S. Nojiri, S. D. Odintsov and M. Sami, Phys. Rev. D 74, 046004 (2006); M. Szydlowski, O. Hrycyna and A. Kurek, Phys. Rev. D 77, 027302 (2008); M. R. Setare and E. N. Saridakis, Phys. Lett. B 671, 331 (2009); M. R. Setare and E. N. Saridakis, JCAP 0903, 002 (2009).

[13] A. R. Liddle and R. J. Scherrer, Phys. Rev. D 59, 023509 (1998); V. Faraoni, Phys. Rev. D 62, 023504 (2000); N. Bartolo and M. Pietroni, Phys. Rev. D 61, 023518 (2000); O. Bertolami and P. J. Martins, Phys. Rev. D 61, 064007 (2000); R. de Ritis, A. A. Marino, C. Rubano and P. Scudellaro, Phys. Rev. D. 62, 043506 (2000); T.D. Saini, S. Raychaudhury, V. Sahni and A. A. Starobinsky, Phys. Rev. Lett. 85, 1162 (2000); G. Gupta, E. N. Saridakis and A. A. Sen, Phys. Rev. D 79, 123013 (2009).
[14] L. Amendola, Phys. Lett. B 301, 175 (1993).

[15] S. Capozziello and G. Lambiase, Gen. Rel. Grav. 31, 1005 (1999); S. Capozziello, G. Lambiase and H. J. Schmidt, Annalen Phys. 9, 39 (2000); S. F. Daniel and R. R. Caldwell, Class. Quant. Grav. 24, 5573 (2007).

[16] A.B. Balakin, H. Dehnen, A.E. Zayats, Phys. Rev. D 76 (2007) 124011; Gen. Rel. Grav. 40 (2008) 2493; Int. J. Mod. Phys. D 17 (2008) 1255.

[17] A.B. Balakin, H. Dehnen, A.E. Zayats, Annals Phys. 323 (2008) 2183.

[18] S. V. Sushkov, Phys. Rev. D 80, 103505 (2009).

[19] E. N. Saridakis and S. V. Sushkov, in preparation.

[20] G. F. R. Ellis and R. Maartens, Class. Quant. Grav. 21, 223 (2004).

[21] L. Baum and P. H. Frampton, Phys. Rev. Lett. 98, 071301 (2007).

[22] R. R. Caldwell, M. Kamionkowski and N. N. Weinberg, Phys. Rev. Lett. 91, 071301 (2003); P. F. Gonzalez-Diaz, Phys. Rev. D 68, 021303 (2003); R. Kallosh, J. Kratochvil, A. Linde, E. Linder and M. Shmakova, JCAP 0310, 015 (2003); S. Nojiri, S. D. Odintsov and S. Tsujikawa, Phys. Rev. D 71, 063004 (2005).

[23] J. Martin, P. Peter, N. Pinto Neto and D. J. Schwarz, Phys. Rev. D 65, 123513 (2002); M. G. Brown, K. Freese and W. H. Kinney, JCAP 0803, 002 (2008); R. Brandenberger, Phys. Rev. D 80, 043516 (2009); Y. F. Cai and E. N. Saridakis, JCAP 0910, 020 (2009).

[24] B. Feng, X. L. Wang and X. M. Zhang, Phys. Lett. B 607, 35 (2005); Z. K. Guo, et al., Phys. Lett. B 608, 177 (2005); M.-Z Li, B. Feng, X.-M Zhang, JCAP, 0512, 002 (2005); B. Feng, M. Li, Y.-S. Piao and X. Zhang, Phys. Lett. B 634, 101 (2006); W. Zhao and Y. Zhang, Phys. Rev. D 73, 123509 (2006); M. R. Setare and E. N. Saridakis, JCAP 0809, 026 (2008); Y. F. Cai, E. N. Saridakis, M. R. Setare and J. Q. Xia, arXiv:0909.2776 [hepth].

[25] R. C. Tolman, Relativity, Thermodynamics and Cosmology (Clarendon Press, Oxford, 1934); P. J. Steinhardt and N. Turok, Phys. Rev. D 65, 126003 (2002); S. Mukherji and M. Peloso, Phys. Lett. B 547 (2002) 297; E. N. Saridakis, Nucl. Phys. B 808, 224 (2009). 